\title{
Molecular evolution of color vision in vertebrates
}

\author{
Shozo Yokoyama* \\ Department of Biology, Biological Research Laboratories, Syracuse University, 130 College Place, Syracuse, NY 13244, USA
}

Received 10 December 2001; received in revised form 4 March 2002; accepted 17 July 2002

\begin{abstract}
Visual systems of vertebrates exhibit a striking level of diversity, reflecting their adaptive responses to various color environments. The photosensitive molecules, visual pigments, can be synthesized in vitro and their absorption spectra can be determined. Comparing the amino acid sequences and absorption spectra of various visual pigments, we can identify amino acid changes that have modified the absorption spectra of visual pigments. These hypotheses can then be tested using the in vitro assay. This approach has been a powerful tool in elucidating not only the molecular bases of color vision, but the processes of adaptive evolution at the molecular level. (C) 2002 Elsevier Science B.V. All rights reserved.
\end{abstract}

Keywords: Visual pigment; Wavelength absorption; Color vision; Vertebrate

\section{Introduction}

Vision has profound effects on the evolution of organisms by affecting survivorship through such behaviors as mating, foraging, and predator avoidance. The data collected by vision scientists over the last century demonstrate beyond any doubt that ecology has been a major factor in directing the evolution of visual systems (Walls, 1942; Lythgoe, 1979; Jacobs, 1981; Bowmaker, 1991; Yokoyama and Yokoyama, 1996). The photosensitive molecules, visual pigments, consist of an apoprotein, opsin, and a chromophore, either 11-cis-retinal or 11-cis-3,4dehydroretinal. Opsin and the chromophore are bound to each other by a Schiff base (Mathies et al., 1976; Palczewski et al., 2000). How did vertebrates modify their color vision to adapt to various environments? This evolutionary question is closely related to a central question in phototransduction: how do visual pigments detect a wide range of wavelengths using the same chromophore? Thus, evolutionary biology and vision science have an important common goal. To explore the molecular bases of spectral tuning of visual pigments, however, we had to wait for two developments. First, in order to conduct genetic analyses of

Abbreviations: $\lambda_{\max }$, wavelength of maximal absorption; RH1, rhodopsin; RH2, RH1-like; SWS1, short wavelength-sensitive type 1; SWS2, SWS type 2; LWS, long wavelength-sensitive; MWS, middle wavelength-sensitive.

* Tel.: + 1-315-443-9166; fax: + 1-315-443-2012.

E-mail address: syokoyam@mailbox.syr.edu (S. Yokoyama). visual pigments, it was necessary to clone opsin genes. This was initiated in 1986 when the opsin genes were cloned from cow and human (Nathans and Hogness, 1983, 1984; Nathans et al., 1986). The availability of these opsin clones led to the isolation of other orthologous and paralogous genes from various species. Second, in the late 1980s, it became possible to express opsins in cultured cells, reconstitute with 11-cis-retinal, and measure the absorption spectra of the resulting visual pigments in vitro (Oprian et al., 1987; Nathans, 1990a,b). These advances in vision science provide a rare opportunity to study the molecular bases of adaptive evolution.

In vertebrates, it is very difficult to find genetic systems where evolutionary hypotheses can be experimentally tested (Golding and Dean, 1998). However, knowing the amino acid sequences and absorption spectra of various visual pigments, we can identify potentially important amino acid changes which may have been responsible for the adaptation of various visual pigments. Importantly, these evolutionary hypotheses can then be tested by constructing the wild-type and mutant pigments and determining if (and how) the amino acids in question actually affect their color detection. In turn, these experiments elucidate the molecular mechanisms of spectral tuning of visual pigments. Here, I shall review the evolutionary patterns of functional differentiation of visual pigments, which represents a group of $G$ protein-coupled receptors (Palczewski et al., 2000). 


\section{Visual pigments}

A striking feature of the visual pigments is the presence of seven stretches of relatively hydrophobic amino acids. This profile is remarkably similar to that of bacteriorhodopsin, a proton pump from Halobacterium halobium, and as a consequence a bacteriorhodopsin-like topology has been proposed (Hargrave et al., 1983). The protein is structurally similar to $\mathrm{G}$ protein-coupled receptors. Indeed, a recent crystallographic analysis of the bovine rhodopsin confirms the existence of seven transmembrane (TM) helices (Palczewski et al., 2000). At present, the amino acid sequences and absorption spectra of over 100 visual pigments have been determined (Yokoyama, 2000a; Ebrey and Koutalos, 2001).

Based on their amino acid sequences, the retinal visual pigments are classified into five paralogous groups: (1) RH1 (rhodopsins), (2) RH2 (RH1-like), (3) short wavelengthsensitive type 1 (SWS1), (4) SWS type 2 (SWS2), and (5) long wavelength- and middle wavelength-sensitive (LWS/MWS) pigments. The RH1, RH2, SWS1, SWS2, and LWS/MWS pigment groups are also known as Rh, M2, S, M, and L groups, respectively (Okano et al., 1992; see also Ebrey and Koutalos, 2001). The RH1 pigments are usually expressed in rods and the other four groups of visual pigments usually in cones. The RH1 pigments are more closely related to RH2, and then to the SWS2, SWS1, and LWS/MWS groups, in that order (Yokoyama and Yokoyama, 1996; Yokoyama, 1997; Yokoyama, 2000a). The five paralogous genes have arisen through four gene duplication events. The RH1 group contains pigments from a wide variety of organisms, ranging from lampreys to mammals. Thus, the most recent gene duplication event of the four occurred prior to the divergence of various vertebrates and the vertebrate ancestor must have possessed all five groups of visual pigments (Yokoyama and Yokoyama, 1996). The functions of these pigments are determined by their wavelengths of maximum absorption, referred to as $\lambda_{\max }$.

The ability of humans to see the wavelength of light ranging in wavelength from $\sim 400$ to 650 nanometers $(\mathrm{nm})$ is controlled by rhodopsins with a $\lambda_{\max }$ of $497 \mathrm{~nm}$ and socalled 'blue', 'green', and 'red' cone pigments with $\lambda_{\max } \mathrm{s}$ of $\sim 420,530$, and $560 \mathrm{~nm}$, respectively (Boynton, 1979). The 'rhodopsin', 'blue', and the 'red and green' pigments in human belong to the RH1, SWS1, and LWS/MWS pigment groups, respectively. So far, neither RH2 nor SWS2 opsin genes has been found in the human and other mammalian genomes. These pigments must have been lost in an early stage of mammalian evolution. When various visual pigments in vertebrates are considered, the RH1, RH2, SWS1, SWS2, and LWS/MWS pigments have $\lambda_{\max } \mathrm{s}$ of 480-510, 470-510, 360-430, 440-460, and 510-560 nm, respectively (Yokoyama and Yokoyama, 1996; Yokoyama, 2000a; Ebrey and Koutalos, 2001).

The cloning and molecular characterization of the opsin genes can be done using standard recombinant DNA methods (Nathans and Hogness, 1983, 1984; Nathans et al., 1986). To determine the $\lambda_{\max } \mathrm{s}$ of visual pigments, we first isolate total RNAs from a retina or whole eye. From this RNA, the opsin cDNAs are obtained by reverse transcription-polymerase chain reaction using appropriate primers, are subcloned into an expression vector, and are expressed in cultured cells (Fig. 1A). These opsins are incubated with 11-cis-retinal in the dark (Fig. 1B). The resulting visual pigments are then purified by immunoaffinity chromatography, in which the chromophore is surrounded by seven TM helices (Fig. 1C; see also
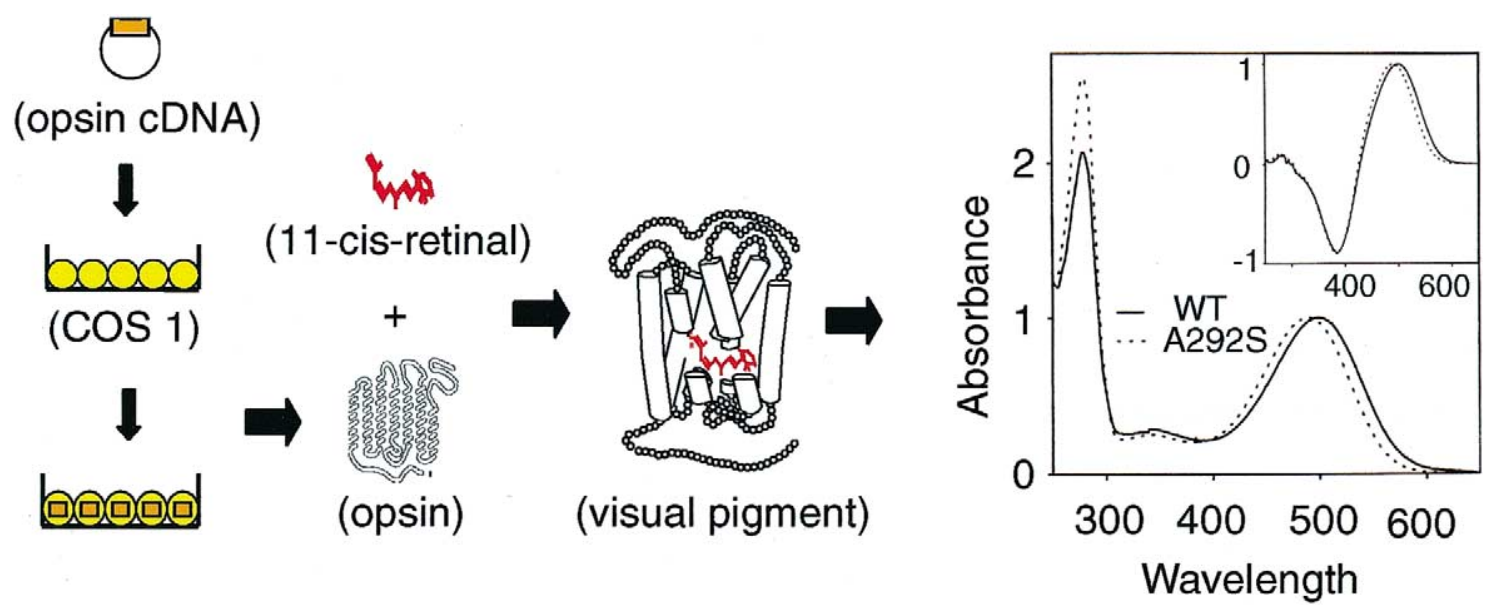

A

B

C

Fig. 1. In vitro assays of the absorption spectra of the wild-type and mutant bovine RH1 pigments. (A) The opsin cDNAs in an expression vector, pMT5, are expressed in COS1 cells by transient transfection. (B) The visual pigments are then regenerated by incubating the opsins with 11-cis-retinal in the dark. (C) The resulting visual pigments are then purified by immunoaffinity chromatography by using monoclonal antibody 1D4 Sepharose 4B. The absorption spectra of the visual pigment are recorded using a spectrophotometer. (D) The amino acid change A292S has been obtained by site-directed mutagenesis. The absorption spectrum in the inset shows the $\lambda_{\max }$ determined by dark-light spectrum. 
Palczewski et al., 2000). The absorption spectra of visual pigments are recorded using a spectrophotometer (Yokoyama, 2000b). As an example, the absorption spectrum of the bovine RH1 pigment measured in the dark (dark spectrum) is shown in Fig. 1D. This spectrum has peaks at 280 and $500 \mathrm{~nm}$ (solid curve, Fig. 1D). The peak of $280 \mathrm{~nm}$ is due to proteins other than structurally stable visual pigments, while the $500 \mathrm{~nm}$ peak shows the $\lambda_{\max }$ of this pigment. When visual pigments are exposed to light (light spectrum), the second peak shifts to $\sim 380 \mathrm{~nm}$, showing that 11-cis-retinal in the pigment is isomerized and all-trans-retinal is formed (Yokoyama, 2000b). The $\lambda_{\max }$ can also be estimated by subtracting the light spectrum from the dark spectrum (inset, Fig. 1D). Using this in vitro assay, we can also evaluate the effects of single and multiple amino acid changes on the shift in the $\lambda_{\max }$. As an example, the effect of a mutation from alanine to serine at site 292 (A292S), which reduces the $\lambda_{\max }$ by $10 \mathrm{~nm}$, is also shown in Fig. 1D, dotted curves. This in vitro assay can be applied to virtually any visual pigment. In the following, we shall consider dim vision, red-green color vision, and ultraviolet (UV) vision, separately. Throughout the paper, the amino acid residue numbers are those of the bovine rhodopsin (Nathans and Hogness, 1983).

\section{The coelacanths and dim vision}

The coelacanths, Latimeria chalumnae, live at a depth of $200 \mathrm{~m}$ near the coast of the Comoros islands in the western Indian Ocean (Fricke et al., 1995; Schliewen et al., 1993). Out of the five groups of visual pigments, the coelacanths have retained only $\mathrm{RH} 1$ and $\mathrm{RH} 2$ pigments, which now have $\lambda_{\max }$ s of 485 and $478 \mathrm{~nm}$, respectively (Yokoyama et al., 1999). Compared to those of most orthologous pigments, these $\lambda_{\max }$ s are reduced by $\sim 10-20 \mathrm{~nm}$. The ocean floor at the depth of $\sim 200 \mathrm{~m}$ receives only a narrow strip of light at around $480 \mathrm{~nm}$ from the surface (Jerlov, 1976). Since the coelacanth is not known for its vertical migration, these blue-shifts in the $\lambda_{\max } \mathrm{s}$ and the loss of the 'blue', 'green', and 'red' pigments may be expected. However, when we realize that the $\lambda_{\max } \mathrm{s}$ of 485 and $478 \mathrm{~nm}$ are devised to visualize the entire spectrum of color available to the coelacanths, the co-adaptation of the two pigments to a benthic environment is simply amazing.

How did the coelacanth achieve these blue-shifts in the $\lambda_{\max }$ ? To explore the molecular bases of this adaptive evolution, let us consider some selected RH1 and RH2 pigments (Fig. 2). The amino acid sequences of the pigments at all ancestral nodes in Fig. 2 are inferred by using a likelihood-based Bayesian method, in which the amino acid replacement models of Jones et al. (1992); Dayhoff et al. (1978), and the equal-input model are considered (Yang et al., 1997). By tracing these ancestral amino acid sequences, we can identify potentially important amino acid replacements in the spectral tuning for each branch. In this way, E122Q/A292S and E122Q/M207L are identified along the branches leading to the coelacanth RH1 and RH2 pigments, respectively (Fig. 2). Amino acid changes Q122E, S292A, and Q122E/S292A in the RH1 pigment increase the $\lambda_{\max }$ by 10,8 , and $26 \mathrm{~nm}$, respectively. Similarly, Q122E, L207M, and Q122E/1207M in the RH2 pigment increase the $\lambda_{\max }$ by 13,6 , and $21 \mathrm{~nm}$, respectively (Yokoyama et al., 1999). Thus, we can explain the blue-shift in the $\lambda_{\max }$ in the RH1 pigment by E122Q/A292S and that of the RH2 pigment by E122Q/M207L. These three amino acids in TM helices III, V, and VII are located close to the chromophore in the center of TM segments (Fig. 3), probably causing significant interactions between them (see also Palczewski et al., 2000).

Recently, another coelacanth has been found off the coast of Indonesia, some $10,000 \mathrm{~km}$ away from the Comoros population (Erdmann et al., 1998). The Indonesian coelacanth also uses the RH1 and RH2 pigments with $\lambda_{\max } \mathrm{s}$ of 485 and $478 \mathrm{~nm}$, respectively, each of which differ at only one amino acid site from the orthologous pigment in the African coelacanth (Yokoyama and Tada, 2000). These observations strongly suggest that the co-adaptation of the two pigments occurred prior to the divergence of the two coelacanth populations.

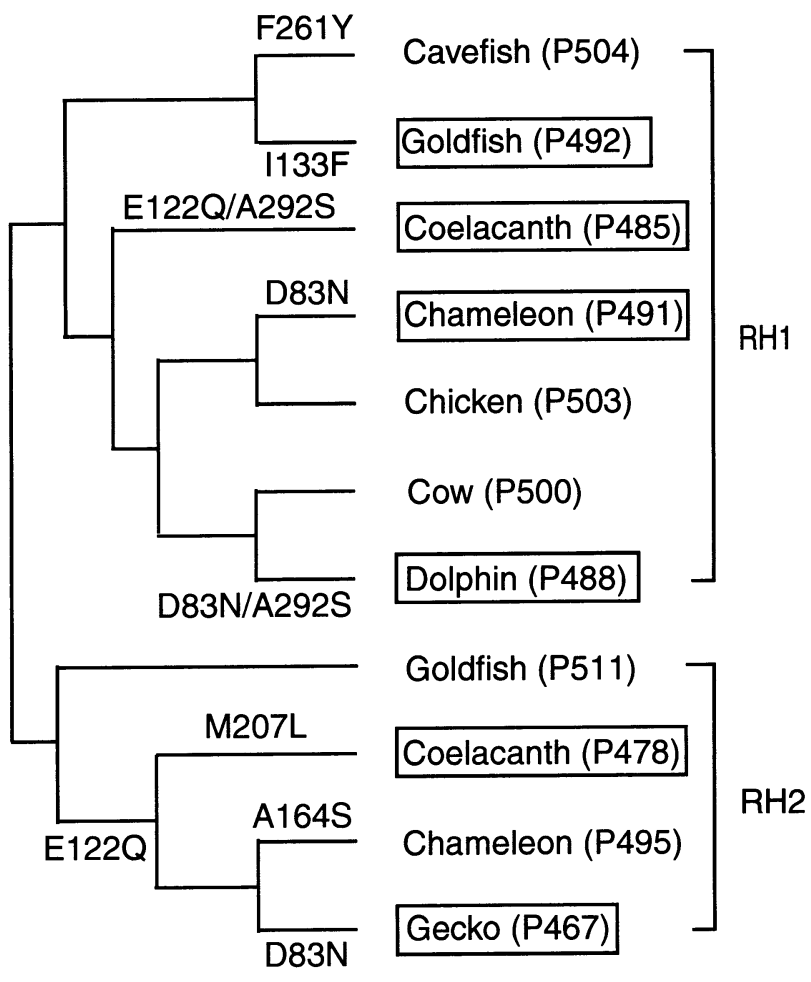

Fig. 2. A composite tree topology of selected RH1 and RH2 pigments and amino acid replacements inferred by a likelihood Bayesian method (Yang et al., 1997). The numbers after P refer to $\lambda_{\max } \mathrm{s}$ of visual pigments. Amino acid replacements are shown next to different branches. The pigments with blue-shifted $\lambda_{\max } \mathrm{s}$ are indicated by rectangles. Cavefish, Astyanax fasciatus; goldfish, Carassius auratus; coelacanth, Latimeria chalumnae; chameleon, Anolis carolinensis; chicken, Gallus gallus; cow, Bos taurus; dolphin, Tursiops truncatus; gecko, Gekko gekko. 


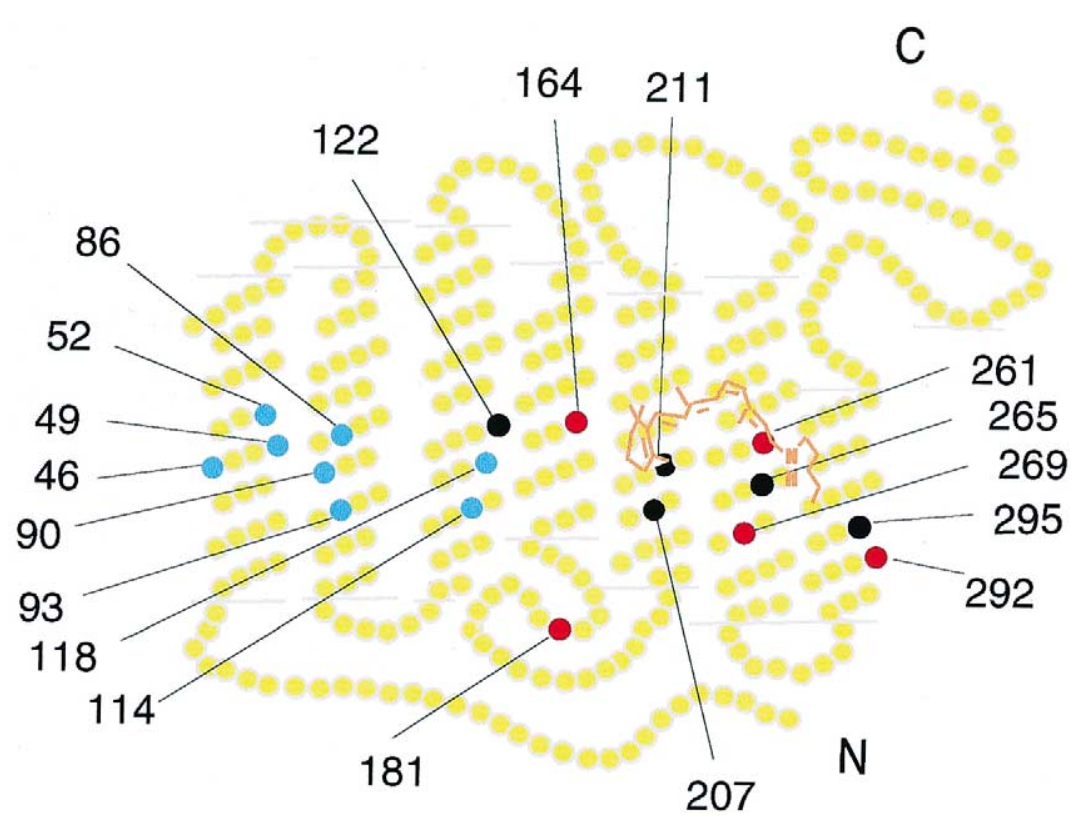

Fig. 3. Secondary structure of bovine RH1 opsin, showing naturally occurring amino acid mutations that cause significant $\lambda_{\max }$-shifts. The model is based on Palczewski et al. (2000). Blue, red and black circles indicate the amino acid sites that are involved in the spectral tuning of SWS1, LWS/MWS, and RH1/RH2 pigments, respectively. The 11-cis-retinal is shown in orange.

The blue-shifts in the $\lambda_{\max } \mathrm{s}$ of RH1 and RH2 pigments are not unique to the coelacanths. Similar examples are also found in such pigments as the RH1 pigments in the goldfish, chameleon, and dolphin and the RH2 pigment in the gecko (Fig. 2). Among these species, dolphins can inhabit an environment where dim and blue light dominate and the blue-shifted $\lambda_{\max }$ can be explained by their adaptation to their habitat. Both chameleon (Provencio et al., 1993) and goldfish (Palacios et al., 1998) use almost exclusively 11cis-3,4-dehydroretinal. The pigment with 11-cis-3,4-dehydroretinal (A2 pigment) usually detects significantly more reddish color than that with 11-cis-retinal chromophore (A1 pigment) (Whitmore and Bowmaker, 1989; Harosi, 1994). The relationship between the $\lambda_{\max }$ of the A1 pigment (L1) and that of the A2 pigment (L2) may be expressed by $\mathrm{L} 2=(\mathrm{L} 1 / 52.5)^{2.5}+250$ (Whitmore and Bowmaker, 1989), or by $\mathrm{L} 2=10^{4} /\left[\left(10^{4} / \mathrm{L} 1\right)-0367-0.050\left\{\left(10^{4} /\right.\right.\right.$ L1) -23.347$\}^{2}$ ] (Harosi, 1994). Thus, it is conceivable that the chameleon and goldfish pigments needed to decrease the $\lambda_{\max }$ so that their dim vision be readjusted to detect light closer to $500 \mathrm{~nm}$. Unlike the evolutionarily closely related chameleon, the gecko has pure-rod retinas (Walls, 1934) and does not use SWS2 pigments for its blue vision. In order to detect light of $\sim 460 \mathrm{~nm}$, the gecko might have replaced SWS2 pigment by RH2 pigment simply because $\mathrm{RH} 2$ pigment was more closely related to the rodspecific RH1 pigment and was easier to adapt to rods.

For the RH1 pigments, D83N and A292S occurred twice independently (Fig. 2). In addition, F261Y occurred in the cavefish pigment, and I133F in the goldfish pigment (Fig. 2). D83N and A292S decrease the $\lambda_{\max }$ of the bovine RH1 pigment by $6 \mathrm{~nm}$ (Nathans, 1990a) and $10 \mathrm{~nm}$ (Fig. 1D), respectively, whereas F261Y increases the $\lambda_{\max } \mathrm{s}$ of the cavefish and bovine RH1 pigments by $\sim 10 \mathrm{~nm}$ (Yokoyama et al., 1995; Chan et al., 1992). Thus, D83N and A292S can explain the blue-shifted $\lambda_{\max } \mathrm{s}$ of the chameleon and dolphin pigments. The effect of I133F on the $\lambda_{\max }$-shift in the goldfish RH1 pigment has not been determined. However, since virtually all SWS1 and SWS2 pigments with $\lambda_{\max } \mathrm{s}$ at $360-460 \mathrm{~nm}$ have $\mathrm{F} 133$, it is possible that $\mathrm{I} 133 \mathrm{~F}$ is responsible for the blue-shift in the $\lambda_{\max }$ of the goldfish RH1 pigment. For the RH2 pigments, E122Q occurred in the tetrapod ancestor, followed by M207L and D83N in the ancestral coelacanth and gecko pigments, respectively, while A164S occurred in the ancestral chameleon pigment (Fig. 2). Since E122Q decreases the $\lambda_{\max }$ of the coelacanth RH2 pigment by $\sim 10 \mathrm{~nm}$, the common ancestor of tetrapod RH2 pigments must have had a more blue-shifted $\lambda_{\max }$ than the ancestral vertebrate RH2 pigment. Thus, the $\lambda_{\max }$ of the chameleon pigment may have been increased by A164S, whereas D83N may have decreased the $\lambda_{\max }$ of the gecko pigment. The actual effects of these amino acid replacements in the goldfish, chameleon, and gecko pigments on the $\lambda_{\max }$-shift, however, remain to be evaluated.

\section{Red-green color vision}

Many MWS and LWS pigments, which together form one group, have $\lambda_{\max } \mathrm{s}$ of $\sim 530$ and $\sim 560 \mathrm{~nm}$, respectively (Yokoyama, 2000a; Ebrey and Koutalos, 2001). It has been proposed that the difference in the color sensitivities between these two groups of pigments is due to three amino acids A164/F261/A269 in the MWS pigments and 
S164/Y261/T269 in the LWS pigments, respectively (Yokoyama and Yokoyama, 1990; Neitz et al., 1991). When A164S/F261Y/A269T are introduced into the bovine RH1 pigment, the $\lambda_{\max }$ of the mutant pigments increase by 2, 10, and $14 \mathrm{~nm}$, respectively (Chan et al., 1992). If we assume that the effects of these changes are additive (see below), then a total of these $\lambda_{\max }$-shifts, $26 \mathrm{~nm}$, explains the $\lambda_{\max }$-difference between the MWS and LWS pigments reasonably well. Quantitatively the same conclusion has been reached by introducing these three and the reverse mutations into the human MWS and LWS pigments, respectively (Asenjo et al., 1994). Three sites 164, 261, and 269 in TM helices interact with the chromophore (Fig. 3). Thus, the 'three-sites' rule (Yokoyama and Radlwimmer, 1998) can explain most differences between the $\lambda_{\max } \mathrm{S}$ of many MWS and LWS pigments. In mammals, however, some exceptions have been found. That is, having essentially LWS pigment-specific amino acids A164/ Y261/T269, the orthologous pigments in mouse, rat, and rabbit have $\lambda_{\max } \mathrm{s}$ of $\sim 510 \mathrm{~nm}$ (Sun et al., 1997; Radlwimmer and Yokoyama, 1998). These extremely blue-shifted $\lambda_{\max }$ a are achieved by two amino acid changes H181Y and A292S (Sun et al., 1997). The site 292 in TM helix VII can interact with the chromophore directly. It is not apparent from Fig. 3, but the site 181 is folded into the TM region (Palczewski et al., 2000). In addition, H181, but not Y181, binds to chloride and H181Y may cause a structural change, resulting in a significant $\lambda_{\max }$-shift (Wang et al., 1993). Thus, to explain the spectral tuning of all LWS/ MWS pigments, it is necessary to consider amino acid differences at five sites, 164, 181, 261, 269, and 292 (Fig. 3).

Multiple regression analyses of 26 currently known LWS/MWS pigments show that the pigment with S164/H181/Y261/T269/A292 has a $\lambda_{\max }$ of $559 \mathrm{~nm}$ and those with mutations S164A, H181Y, Y261F, T269A, A292S, and S164A/H181Y shift the $\lambda_{\max }$ by $-7,-28,-8$, $-15,-27$, and $11 \mathrm{~nm}$, respectively (Yokoyama and Radlwimmer, 2001). Based on the amino acid composition at the five sites, these 26 contemporary pigments can be classified into ten groups (Table 1). The effects of amino acid differences at the remaining 359 sites on the $\lambda_{\max }$-shift have been tested in more detail by considering the common ancestor of the human, marmoset, rabbit, goat, deer, guinea pig, squirrel, and mouse pigments. In this inference, we have considered the phylogenetic relationship of ((() human LWS, human MWS), (marmoset $\mathrm{LWS}_{1}$, marmoset $\mathrm{LWS}_{2}$, marmoset MWS)), rabbit, (goat, deer)), guinea pig, (squirrel, mouse)), where $\mathrm{LWS}_{1}, \mathrm{LWS}_{2}$, and MWS of the marmoset pigments show the $\lambda_{\max } \mathrm{s}$ of 561, 553, and 539 $\mathrm{nm}$, respectively. The amino acid sequence of their common ancestral pigment $\mathrm{X}$ was first inferred by using the likelihood-based Bayesian method, as before. The pigment $\mathrm{X}$ and the contemporary pigments can differ considerably at the 359 background sites. For example, it differs from the human LWS pigment at 27 sites, while it differs from the cavefish LWS pigment at 91 sites. When it is compared to the 26 contemporary pigments, pigment $\mathrm{X}$ differs at most three of five critical amino acid sites (Table 1). The mole rat pigment is identical to pigment $\mathrm{X}$ at the five critical sites.

Pigment $\mathrm{X}$ was actually constructed by introducing the necessary amino acid changes into the closely related squirrel and rabbit opsin cDNAs, and recombining them. Then, the nine mutant pigments were also constructed by introducing 1-3 amino acid changes into pigment $X$. In vitro assays of pigment $\mathrm{X}$ and the nine mutant pigments show that, as long as the amino acid changes at the five sites are the same, their $\lambda_{\max } \mathrm{s}$ are identical to those of the corresponding contemporary pigments (Table 1). This demonstrates that amino

Table 1

The $\lambda_{\max }$ of pigment $\mathrm{X}$ and those of pigment $\mathrm{X}$ with amino acid changes (underlined) at sites 164, 181, 261, 269, and 292

\begin{tabular}{|c|c|c|c|c|c|c|c|}
\hline & \multirow[t]{2}{*}{ Pigment $^{\mathrm{a}}$} & \multicolumn{5}{|c|}{ Amino acids at } & \multirow{2}{*}{$\begin{array}{l}\text { Pigment } \mathrm{X} \\
\lambda_{\max }(\mathrm{nm})\end{array}$} \\
\hline & & 164 & 181 & 261 & 269 & 292 & \\
\hline 0 . & The mammalian ancestor $(\mathrm{X})$ & A & $\mathrm{Y}$ & $\mathrm{Y}$ & $\mathrm{T}$ & A & $533 \pm 1$ \\
\hline 1. & Mole rat (P534) & A & $\mathrm{Y}$ & $\mathrm{Y}$ & $\mathrm{T}$ & A & \\
\hline 2. & Human (P552), marmoset (P553), goat (P553), cat (P553) & A & $\underline{\mathrm{H}}$ & $\mathrm{Y}$ & $\mathrm{T}$ & A & $533 \pm 1$ \\
\hline 3. & $\begin{array}{l}\text { Human (P560), marmoset (P561), chicken (P561), pigeon (P559), zebra finch (P559), } \\
\text { clawed frog (P557), chameleon (P560), cavefish (P558), goldfish (P559) }\end{array}$ & $\underline{\mathrm{S}}$ & $\underline{\mathrm{H}}$ & $\mathrm{Y}$ & $\mathrm{T}$ & A & $557 \pm 2$ \\
\hline 4. & Human (P530), deer (P531), gecko (P527), cavefish (P530) & A & $\underline{\mathrm{H}}$ & $\underline{\mathrm{F}}$ & $\underline{\mathrm{A}}$ & A & $530 \pm 2$ \\
\hline 5 . & Marmoset (P539) & A & $\underline{\mathrm{H}}$ & Y & $\underline{\mathrm{A}}$ & A & $537 \pm 2$ \\
\hline 6. & Mouse (P509), rat (P508), rabbit (P509) & A & $\mathrm{Y}$ & Y & $\mathrm{T}$ & $\underline{S}$ & $509 \pm 1$ \\
\hline 7. & Guinea pig (P516) & A & $\mathrm{Y}$ & Y & $\underline{\mathrm{A}}$ & $\bar{A}$ & $519 \pm 1$ \\
\hline 8. & Squirrel (P532) & $\underline{\mathrm{S}}$ & $\mathrm{Y}$ & Y & $\overline{\mathrm{T}}$ & A & $532 \pm 1$ \\
\hline 9. & Dolphin (P524) & A & $\underline{\mathrm{H}}$ & $\mathrm{Y}$ & $\mathrm{T}$ & $\underline{S}$ & $523 \pm 1$ \\
\hline 10. & Horse (P545) & A & $\underline{\mathrm{H}}$ & $\underline{F}$ & $\mathrm{~T}$ & A & $545 \pm 1$ \\
\hline
\end{tabular}

\footnotetext{
a The number after P refers to $\lambda_{\max }$ determined by in vitro assay. Cat: Felis catus; cave fish: Astyanax fasciatus; chameleon: Anolis carolinensis; chicken: Gallus gallus; deer: Odocoileus virginianus; dolphin: Tursiops truncatus; frog: Xenopus laevis; gecko: Gekko gekko; goat: Capra hircus; goldfish: Carassius auratus; guinea pig: Cavia porcellus; horse: Equus caballus; human: Homo sapiens; marmoset: Callithrix jacchus; mole rat: Spalax ehrenbergi; mouse: Mus musculus; pigeon: Columba livia; rabbit: Oryctolagus cuniculus; rat: Rattus norvegicus; squirrel: Sciurus carolinensis; zebra finch: Taeniopygia guttata. Modified from Yokoyama and Radlwimmer (2001).
} 
acid changes at the background sites are not important in the spectral tuning in the LWS/MWS pigments. Indeed, the 'five-sites' rule fully explains the variable $\lambda_{\max } \mathrm{s}$ of the contemporary pigments (Yokoyama and Radlwimmer, 2001). Next, by considering the phylogenetic relationship of ((((human LWS, human MWS), goat, (squirrel, mouse)), ((chameleon, goat), chicken), clawed frog), (cavefish LWS, cavefish MWS)), the amino acid sequences of their ancestral pigments have been inferred and constructed (Yokoyama and Radlwimmer, 2001). Most of these ancestral pigments have S164/H181/Y261/T269/A292, just like the group 3 pigments in Table 1, and have $\lambda_{\max } \mathrm{s}$ of $558-563 \mathrm{~nm}$, which are again explained fully by the 'five-sites' rule (Yokoyama and Radlwimmer, 2001).

Thus far, the discussion has been limited to LWS/MWS pigments. In order to appreciate fully the red-green color vision, however, we need to consider additional features of visual pigments as well as photoreceptor cells. That is, many fishes, amphibians, and reptiles use A2 pigments (Walls, 1942). Many fishes have both MWS and LWS pigments. Their A1 pigments with MWS and LWS opsins have $\lambda_{\max } \mathrm{S}$ of $\sim 530$ and $\sim 560 \mathrm{~nm}$, respectively. In nature, however, the corresponding A2 pigments actually detect light at $\sim 560$ and $\sim 620 \mathrm{~nm}$ (Palacios et al., 1998). Furthermore, no MWS pigment has been found in birds and reptiles (Yokoyama, 2000a; Ebrey and Koutalos, 2001). How do these species' pigments detect light maximally at $\sim 530$ $\mathrm{nm}$ ? Interestingly, the RH2 pigments are used for that purpose. For example, A1 pigment with a goldfish RH2 opsin has a $\lambda_{\max }$ of $511 \mathrm{~nm}$ (Fig. 2), but the corresponding A2 pigment has a $\lambda_{\max }$ of $537 \mathrm{~nm}$ (Palacios et al., 1998). The A1 pigment with a chicken RH2 opsin has a $\lambda_{\max }$ of $508 \mathrm{~nm}$ (Okano et al., 1992). Having a green oil-droplet, however, the chicken cone with the RH2 pigments actually has a $\lambda_{\max }$ of $533 \mathrm{~nm}$ (Bowmaker and Knowles, 1977).

Having neither 11-cis-3,4-dehydroretinal nor colored oil droplets, the red-green color vision of mammals is exclusively controlled by their LWS/MWS pigments. In higher primates, the red-green color vision evolved in two different ways. For their red-green color vision, hominoid and Old World monkeys use LWS and MWS opsins, encoded by two separate X-linked loci (Nathans et al., 1986). Most New World monkeys, however, have one corresponding X-linked locus with three alleles (Jacobs, 1981; Bowmaker, 1991). For example, the marmoset has three allelic LWS/MWS pigments with $\lambda_{\max } \mathrm{s}$ of 539, 553, and $561 \mathrm{~nm}$ (Table 1). In these species, therefore, all males are 'red-green color blind', whereas females are either 'color blind' or have complete red-green color vision depending on the genotype.

\section{Ultraviolet (UV) vision}

Many fishes, amphibians, reptiles, birds, and some mammals use UV vision for such basic activities as foraging
(Burkhardt, 1982; Viitala et al., 1995) and mate choice (Bennett et al., 1996, 1997). These species detect light maximally at $360-370 \mathrm{~nm}$ by using UV pigments. The UV pigments and violet (or blue) pigments, with $\lambda_{\max } \mathrm{s}$ of 390$430 \mathrm{~nm}$, belong to the SWS1 group. These $\lambda_{\max } \mathrm{s}$ are modified neither by 11-cis-3,4-dehydroretinal (Ma et al., 2001) nor by oil-droplets (Bowmaker and Knowles, 1977) and UV vision is achieved through UV pigments. When the amino acid sequences of various SWS1 pigments are compared, no common amino acid can be found among the UV pigments, suggesting that the spectral tunings in the UV pigments of various vertebrates have been achieved by different mechanisms. This complication has been overcome by studying avian and non-avian pigments separately.

The zebra finch, canary, and budgerigar SWS1 pigments have $\lambda_{\max } \mathrm{s}$ of $358-366 \mathrm{~nm}$, whereas the orthologous pigments of pigeon and chicken have $\lambda_{\max } \mathrm{s}$ of 393 and $415 \mathrm{~nm}$, respectively, and are violet-sensitive (Fig. 4). By comparing the amino acid sequences of all SWS1 pigments, it has been found that C90 in TM helix II (Fig. 3) is common only to the three avian UV pigments, whereas all other SWS1 pigments, including the chicken and pigeon violet pigments, have S90. Thus, it is highly likely that S90C is responsible for the evolution of the UV pigments in birds. Indeed, the zebra finch and budgerigar pigments with the single mutation C90S attain $\lambda_{\max } \mathrm{s}$ at $397 \mathrm{~nm}$ (Yokoyama et al., 2000) and $398 \mathrm{~nm}$ (Wilkie et al., 2000), respectively. On the other hand, the pigeon and chicken violet pigments with the reverse mutation, S90C, become UV-sensitive (Yokoyama et al., 2000).

To study the molecular bases of UV vision in other vertebrates, the mouse and human SWS1 pigments have been compared. The mouse and human SWS1 pigments have $\lambda_{\max } \mathrm{s}$ of $359 \mathrm{~nm}$ (Yokoyama et al., 1998) and $414 \mathrm{~nm}$ (Fasick et al., 1999), respectively, and differ at 50 amino acid sites. By constructing a series of chimeric mutants between the two pigments, the cause for their $\lambda_{\max }$ difference is traced to 19 amino acid sites in TM helices I-III (Yokoyama and Shi, 2000; Shi et al., 2001). When these 19 amino acids of the mouse pigment are replaced singly by the corresponding amino aids of the human pigment, none of them shifts the $\lambda_{\max }$ from $359 \mathrm{~nm}$. This result shows that the functional differentiation of the UV and violet pigments is achieved by synergistic interactions at multiple amino acid sites in TM helices I-III. Through comparative sequence analyses, eight potentially important amino acids in TM helices I-III are suspected to cause the differentiation of UV and violet (or blue) pigments (Yokoyama and Shi, 2000). In fact, the mouse UV pigment with seven of these amino acid changes, F46T/F49L/T52F/ F86L/T93P/A114G/S118T, achieves a $\lambda_{\max }$ at $411 \mathrm{~nm}$, which is very close to the $\lambda_{\max }$ of the human blue pigment. On the other hand, the human pigment with the reverse mutations achieves a $\lambda_{\max }$ at $360 \mathrm{~nm}$, which is identical to that of the mouse pigment.

Combining the mutagenesis analyses of the avian and 


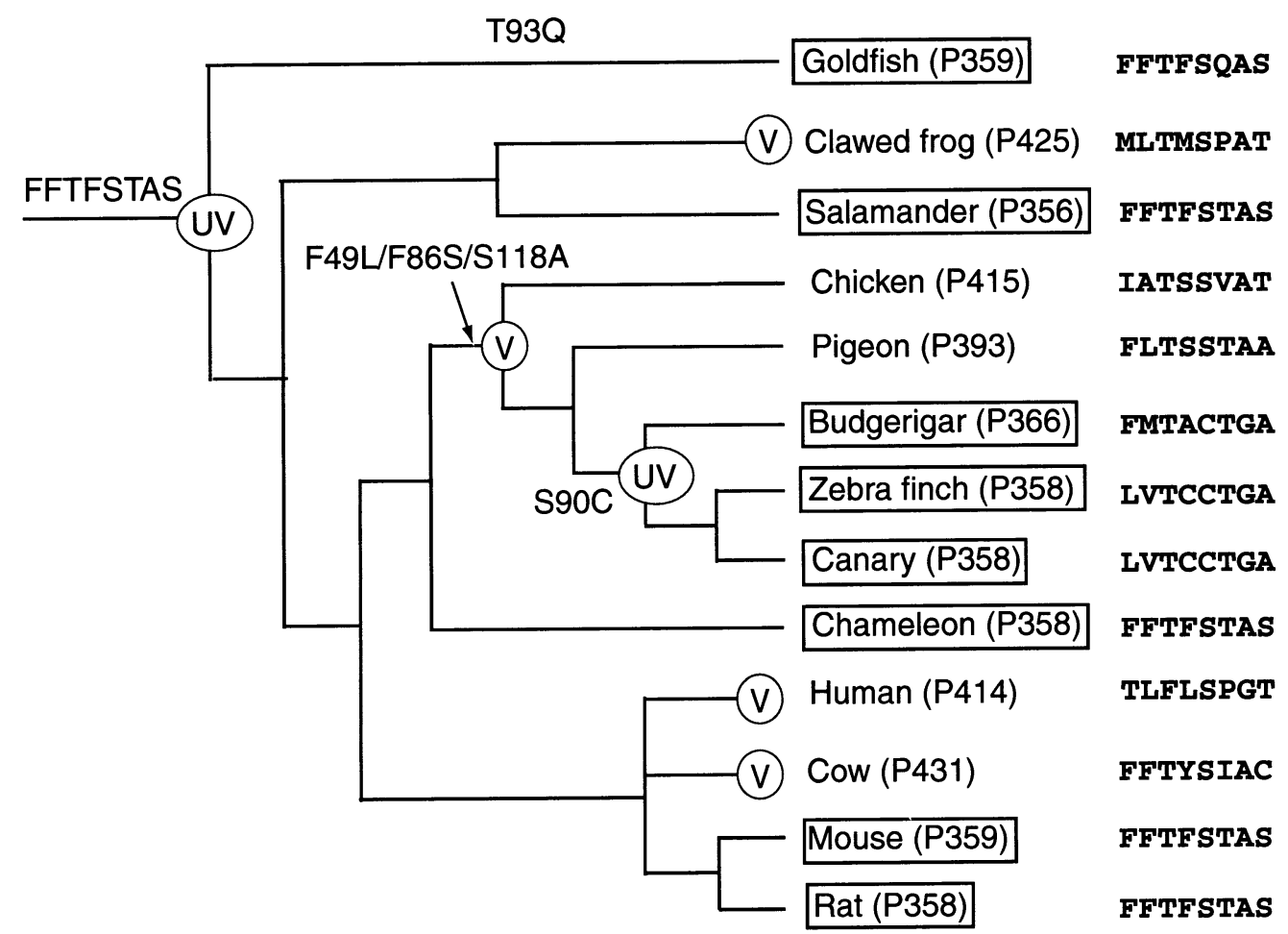

Fig. 4. A composite tree topology of selected SWS1 pigments and critical amino acid replacements at sites, 46, 49, 52, 86, 90, 3, 114, and 118, along the goldfish and avian branches. The UV pigments are boxed. UV and V indicate UV and violet sensitivities, respectively. Amino acid replacements next to the goldfish and avian branches show those at the eight critical sites. The eight letters next to the vertebrate ancestor and 13 contemporary pigments show the eight critical amino acids. Clawed frog, Xenopus laevis; pigeon, Columba livia; budgerigar, Melopsittacus undulatus; zebra finch, Taeniopygia guttata; human, Homo sapiens; mouse, Mus musculus; rat, Rattus norvegicus; and salamander, Ambystoma tigrinum. For other species, see Fig. 2.

mammalian SWS1 pigments, it is likely that the difference between UV and violet pigments are based on a total of eight amino acid sites 46, 49, 52, 86, 90, 93, 114, and 118 (Fig. 3). When the amino acid sequences of the ancestral pigments of the 13 contemporary SWS1 pigments in Fig. 4 are inferred as before, the pigment of the vertebrate ancestor has amino acids F46/F49/T52/F86/S90/T93/A114/S118. This amino acid composition is identical to those of the salamander, chameleon, mouse, and rat UV pigments, but it differs at one site from that of the goldfish UV pigment (Fig. 4). Since the goldfish UV pigment with Q93T does not shift the $\lambda_{\max }$ from that of the wild-type pigment (Yokoyama and Shi, 2000), the ancestral pigment must have had a $\lambda_{\max }$ of $\sim 360 \mathrm{~nm}$ and the fish, salamander, chameleon, mouse, and rat pigments have maintained their UV-sensitivities through purifying selection (Shi et al., 2001).

Fig. 4 also reveals how this ancestral UV pigment has been modified into the modern pigments. In the avian lineage, three amino acid replacements, F49L/F86S/S118A, occurred first in the common ancestor. The amino acid composition at the eight critical sites of this ancestral avian pigment is identical to that of the pigeon violet pigment and, therefore, the ancestral pigment must have been violetsensitive with a $\lambda_{\max }$ of $\sim 395 \mathrm{~nm}$. The ancestor(s) of the budgerigar, zebra finch, and canary pigments then regained UV vision with a single amino acid change, S90C. The human pigment achieved violet-sensitivity by F46T/F49L/
T52F/F86L/T93P/A114G/S118T. Since the divergence from the ancestral UV pigment, the clawed frog, chicken, and bovine violet pigments have accumulated F46M/F49L/ F86M/T93P/S118T, F46I/F49A/F86S/T93V/S118T, and F86Y/T93I/S118C, respectively. Thus, the amino acid compositions at the eight critical sites of various violet pigments can be very different. At present, it is not known which of these amino acid changes are responsible for the change from the ancestral UV pigment to violet pigments.

We have seen that none of the 19 single amino acid changes causes the $\lambda_{\max }$-shift in the mouse UV pigment. Then, why is a single amino acid change S90C (or C90S) effective in making the avian pigment UV- (or violet-) sensitive? Interestingly, when S90C is introduced into the contemporary mouse UV pigment, $\lambda_{\max }$ is unchanged (Shi et al., 2001). The major difference between the mouse and ancestral avian pigments is that the former pigment has maintained the ancestral amino acids at the eight critical sites, whereas the latter pigment has accumulated three amino acid changes at 49, 86, and 118 (Fig. 4). Thus, it is most likely that S90C and C90S in the avian pigments can shift the $\lambda_{\max }$ through their interactions with some or all of the three accumulated mutations. Importantly, most contemporary UV pigments in vertebrates must have maintained their UV-sensitivities by accumulating no more than one of the eight specific amino acid changes. On the other hand, it is likely that violet (or blue) vision evolved from 
UV vision by the replacement of at least two out of the eight critical amino acids in the ancestral UV pigment (Yokoyama and Shi, 2000; Shi et al., 2001). The non-linear effect of amino acid changes on the spectral tuning in the UV pigment is in sharp contrast to approximate additive effects of the amino acid changes on the $\lambda_{\max }$-shift detected in the RH1 (Chan et al., 1992; Lin et al., 1998; Fasick and Robinson, 1998; Yokoyama et al., 1999), RH2 (Yokoyama et al., 1999), and LWS/MWS pigments (Asenjo et al., 1994; Yokoyama and Radlwimmer, 1998, 1999, 2001).

\section{Conclusions and perspectives}

Studies on the structure and function of rhodopsins by Doi et al. (1990) and the subsequent series of papers by H.G. Khorana and his colleagues have dramatically improved our understanding of the roles of key amino acids in visual pigments (see also Sakmar et al., 1989; Zhukovsky and Oprian, 1989; Nathans, 1990a,b; Weitz and Nathans, 1993). Most of the mutations considered in these studies, however, are not found in nature and their significance in the spectral tuning of visual pigments is not immediately clear. Using actual polymorphism data, 18 amino acid changes that cause significant $\lambda_{\max }$-shifts in visual pigments have been identified (Fig. 3). The mutagenesis experiments at the sites 211, 265, and 295 in Fig. 3 were initiated because of amino acid polymorphisms among paralogous visual pigments. These results also need to be interpreted with caution. Note that G90S in bovine RH1 pigment decreases the $\lambda_{\max }$ by $11 \mathrm{~nm}$ (Lin et al., 1998), whereas S90G in the human SWS1 pigment also decreases the $\lambda_{\max }$ by the same extent (Fasick et al., 1999). Clearly, identical amino acid changes can shift the $\lambda_{\max }$ of paralogous visual pigments in opposite directions. The magnitudes of the $\lambda_{\max }$-shift caused by same amino acid changes can also differ even among different orthologous pigments (Yokoyama, 2000a).

Amino acid differences at sites 46, 49, 52, 86, 90, 93, 114, and 118 in TM helices I-III are responsible for the functional divergence of UV and violet vision, whereas those at sites 164, 181, 261, 269, and 292 in TM helices IVVII are responsible for red-green color vision (Fig. 3). The crystal structure of the bovine rhodopsin (RH1) pigment (Palczewski et al., 2000) shows that TM helices I-III and VII are located near the Schiff base nitrogen of the chromophore and TM helices IV-VI are near the ring portion of the chromophore. In particular, the clustering of the eight amino acid sites of the SWS1 pigments at the Schiff base pocket may have an important implication in the spectral tuning in the UV pigments. The Schiff base of the chromophore is usually protonated by E113 in the TM helix III (Sakmar et al., 1989; Zhukovsky and Oprian, 1989; Nathans, 1990a,b). The free protonated Schiff base in solution has a $\lambda_{\max }$ of $440 \mathrm{~nm}$ (Kito et al., 1968). Interacting with an opsin, however, the Schiff base-linked chromophore in a visual pigment can have a $\lambda_{\max }$ from 360 to $635 \mathrm{~nm}$
(Kochendoerfer et al., 1999). On the other hand, the unprotonated Schiff base-linked chromophore in solution has a $\lambda_{\max }$ of $365 \mathrm{~nm}$ (Ball et al., 1949).

How do visual pigments acquire the unprotonated Schiff base? It has been observed that removal of water molecules from the Schiff base pocket can result in displacement of positive charge away from the Schiff base nitrogen, leading to deprotonation of the Schiff base (Rafferty and Shichi, 1981; Deng et al., 1994; Harosi and Sandorfy, 1995; Nagata et al., 1997). Thus, some (or all) of the eight critical amino acids in the UV pigments may eliminate water molecules in the Schiff base pocket. The strong synergistic interactions in the SWS1 pigments may occur because of the highly limited access of water molecules to the Schiff base pocket of the UV pigments (Yokoyama et al., 2000; Shi et al., 2001). Thus, UV pigments may have an unprotonated Schiff baselinked chromophore (Kakitani et al., 1985; Fahmy and Sakmar, 1993; Vought et al., 1999; Yokoyama et al., 2000; Shi et al., 2001; Babu et al., 2001). To elucidate the mechanisms of UV vision, this possibility needs to be explored.

Comparative sequence analyses are useful in identifying potentially important amino acid changes which may be responsible for the spectral tuning in various visual pigments. These evolutionary hypotheses can be tested by in vitro assays. This approach is proven to be effective in elucidating not only the molecular bases of color vision, including red-green color vision and UV vision, but also the molecular bases of the adaptation of organisms to different environments, as exemplified by the coelacanth evolution. At present, the molecular basis of the spectral tuning in visual pigments is best understood for the LWS/MWS pigments, where the 'five-sites' rule has been established. We have also seen that the $\lambda_{\max } \mathrm{s}$ of UV pigments in the SWS1 group are controlled mainly by a total of 8 amino acid sites. However, the exact nature of non-additive effects on the $\lambda_{\max }$-shift of various amino acid replacements in the SWS1 pigments is not known. In order to explore the evolutionary processes of the five groups of visual pigments, it is important to identify the amino acid replacements that are responsible for causing the variable $\lambda_{\max } \mathrm{s}$ of the RH1, RH2, SWS1, and SWS2 pigments. The analyses of the LWS/MWS pigments (Yokoyama and Radlwimmer, 2001) show how we can study the molecular bases and evolutionary process of the spectral tuning of these visual pigments simultaneously.

As more amino acid sequences and absorption spectra of visual pigments accumulate, the evolutionary prediction of potentially important amino acid changes will improve. In the future, the sampling of visual pigments from various photic environments or those associated with different behavioral characteristics would be of particular interest because we may also uncover previously unknown mechanisms of spectral tuning in visual pigments, as we witnessed in elucidating the LWS/MWS pigments. The successes of the comparative sequence analyses followed by 
the mutagenesis experiments demonstrate that the evolutionary approach is a powerful method in enhancing our understanding of the molecular bases of a wide variety of visual pigments. Once molecular bases of spectral tuning of various visual pigments are elucidated, we will be in a much better position to fully appreciate the mechanisms by which the complexity of colors and patterns exhibited by animals and plants are perceived, and how these mechanisms evolved.

\section{Acknowledgements}

Comments by P. Dunham and R. Yokoyama were greatly appreciated. This work was supported by NIH grant R01 GM42379.

\section{References}

Asenjo, A.B., Rim, J., Oprian, D.D., 1994. Molecular determination of human red/green color discrimination. Neuron 12, 1131-1138.

Babu, K.R., Dukkipati, A., Birge, R.R., Knox, B.E., 2001. Regulation of phototransduction in short-wavelength cone visual pigment via the retinylidene Schiff base counterion. Biochemistry 40, 13760-13766.

Ball, S., Collins, F.D., Dalvi, P.D., Morton, R.A., 1949. Studies in vitamin A. 11. Reactions of retinene1 with amino compounds. Biochem. J. 45, 304-307.

Bennett, A.T.D., Cuthill, I.C., Partridge, J.C., 1996. Ultraviolet vision and mate choice in zebra finches. Nature 380, 433-435.

Bennett, A.T.D., Cuthill, I.C., Partridge, J.C., 1997. Ultraviolet plumage colors predict mate preferences in starlings. Proc. Natl. Acad. Sci. USA 94, 8618-8621.

Bowmaker, J.K., 1991. Evolution of photoreceptor and visual pigments. In: Cronly, J.R., Gregory, R.L. (Eds.), Evolution of the Eye and Visual Pigments, CRC Press, Boca Raton, FL, pp. 63-81.

Bowmaker, J.K., Knowles, A., 1977. The visual pigments and oil droplets of the chicken retina. Vision Res. 17, 755-764.

Boynton, R.M., 1979. Human Color Vision, Holt, Rinehart and Winston, New York.

Burkhardt, D., 1982. Birds, berries and UV. Naturwissenschaften 69, $153-157$.

Chan, T., Lee, M., Sakmar, T.P., 1992. Introduction of hydroxyl-bearing amino acid causes bathochromatic spectral shifts in rhodopsin: amino acid substitutions responsible for red-green color pigment spectral tuning. J. Biol. Chem. 267, 9478-9480.

Dayhoff, M.O., Schwartz, R.M., Orcutt, B.C., 1978. A model of evolutionary change in proteins. In: Dayhoff, M.O., (Ed.), Atlas of Protein Sequence and Structure, National Biomedical Research Foundation, Washington, DC, pp. 345-352.

Deng, H., Huang, L., Callender, R., Ebrey, T., 1994. Evidence for a bound water molecule next to the retinal Schiff base in bacteriorhodopsin and rhodopsin: A resonance Raman study of the Schiff base hydrogen/ deuterium exchange. Biophys. J. 66, 1129-1136.

Doi, T., Molday, R.S., Khorana, H.G., 1990. Role of the intradiscal domain in rhodopsin assembly and function. Proc. Natl. Acad. Sci. USA 87, 4991-4995.

Ebrey, T., Koutalos, Y., 2001. Vertebrate photoreceptors. Prog. Ret. Eye Res. 20, 49-94.

Erdmann, M.V., Caldwell, R.I., Moosa, M.K., 1998. Indonesian 'king of the sea' discovered. Nature 395, 335.

Fahmy, K., Sakmar, T.P., 1993. Light-dependent transduction activation by an ultraviolet-absorbing rhodopsin mutant. Biochemistry 32, 9165-9171.

Fasick, J.L., Robinson, P.R., 1998. Mechanism of spectral tuning in the dolphin visual pigment. Biochemistry 37, 432-438.

Fasick, J.L., Lee, N., Oprian, D.D., 1999. Spectral tuning in the human blue cone pigment. Biochemistry 38, 11593-11596.

Fricke, H., Hissmann, K., Schaner, J., Plante, R., 1995. Yet more danger for coelacanths. Nature 374, 314.

Golding, G.B., Dean, A.M., 1998. The structural basis of molecular adaptation. Mol. Biol. Evol. 15, 355-369.

Hargrave, P.A., McDowell, J.H., Curtis, D.R., Wang, J.K., Juszczak, E., Fong, S.-L., Mohana Rao, J.K., Argos, P., 1983. The structure of bovine rhodopsin. Biophys. Struct. Mech. 9, 235-244.

Harosi, F.I., 1994. Analysis of two spectral properties of vertebrate visual pigments. Vision Res. 34, 1359-1367.

Harosi, F.I., Sandorfy, C., 1995. Retinylidene-opsin Schiff base chromophore and their accessibility to water. Photochem. Photobiol. 61, 510-517.

Jacobs, G.H., 1981. Comparative Color Vision, Academic Press, New York.

Jerlov, N.G., 1976. Marine Optics, Elsevier, Amsterdam.

Jones, D.T., Taylor, W.R., Thornton, J.M., 1992. The rapid generation of mutation data matrices from protein sequences. Comput. Appl. Biosci. $8,275-282$.

Kakitani, H., Kakitani, T., Rodman, H., Honig, B., 1985. On the mechanism of wavelength regulation in visual pigments. Photochem. Photobiol. 41, 471-479.

Kito, Y., Suzuki, T., Azuma, M., Sekoguchi, Y., 1968. Absorption spectrum of rhodopsin denatured with acid. Nature 218, 955-957.

Kochendoerfer, G.G., Lin, S.W., Sakmar, T.P., Mathies, R.A., 1999. How color visual pigments are tuned. Trends Biochem. Sci. 24, 300-305.

Lin, S.W., Kochendoerfer, G.K., Carroll, K.S., Wang, D., Mathies, R.A., Sakmar, T.P., 1998. Mechanisms of spectral tuning in blue cone visual pigments: Visible and Raman spectroscopy of blue-shifted rhodopsin mutants. J. Biol. Chem. 273, 24853-24892.

Lythgoe, J.N., 1979. The Ecology of Vision, Clarendon, Oxford.

Ma, J.-X., Kono, M., Xu, L., Das, J., Ryan, J.C., Hazard, E.S., Oprian, D.D., Crouch, R.K., 2001. Salamander UV cone pigment: Sequence, expression, and spectral properties. Vis. Neurosci. 18, 393-399.

Mathies, R., Oseroff, A.R., Stryer, L., 1976. Rapid-flow resonance Raman spectroscopy of photolabile molecules: rhodopsin and isorhodopsin. Proc. Natl. Acad. Sci. USA 73, 1-5.

Nagata, T., Terakita, A., Kandori, H., Kojima, D., Shichida, Y., Maeda, A., 1997. Water and peptide backbone structure in the active center of bovine rhodopsin. Biochemistry 36, 6164-6170.

Nathans, J., 1990a. Determinants of visual pigment absorbance: role of changed amino acids in the putative transmembrane segments. Biochemistry 29, 937-942.

Nathans, J., 1990b. Determinants of visual pigment absorbance: identification of the retinylidene Schiff's base counterion in bovine rhodopsin. Biochemistry 29, 9746-9752.

Nathans, J., Hogness, D.S., 1983. Isolation, sequence analysis, and intronexon arrangement of the gene encoding bovine rhodopsin. Cell 34 , 807-814.

Nathans, J., Hogness, D.S., 1984. Isolation and nucleotide sequence of the gene encoding human rhodopsin. Proc. Natl. Acad. Sci. USA 81, $4851-4855$.

Nathans, J., Thomas, D., Hogness, D.S., 1986. Molecular genetics of human color vision: the genes encoding blue, green, and red pigments. Science 232, 193-201.

Neitz, M., Neitz, J., Jacobs, G.H., 1991. Spectral tuning of pigments underlying red-green color vision. Science 252, 971-974.

Okano, T., Kojima, D., Fukada, Y., Schichida, Y., Yoshizawa, T., 1992. Primary structures of chicken cone visual pigments: Vertebrate rhodopsins have evolved out of cone visual pigments. Proc. Natl. Acad. Sci. USA 89, 5932-5936.

Oprian, D.D., Molday, R.S., Kaufman, R.J., Khorana, H.G., 1987. 
Expression of a synthetic bovine rhodopsin gene in monkey kidney cells. Proc. Natl. Acad. Sci. USA 84, 8874-8878.

Palacios, A.G., Varela, F.J., Srivastava, R., Goldsmith, T.J., 1998. Spectral sensitivity of cones in the goldfish, Carassius auratus. Vision Res. 38, $2135-2146$.

Palczewski, K., Kumasaka, T., Hori, T., Behnke, C.A., Motoshima, H., Fox, B.A., Trong, I.L., Teller, D.C., Okada, T., Stenkamp, R.E., Yamamoto, M., Miyano, M., 2000. Crystal structure of rhodopsin: A G proteincoupled receptor. Science 289, 739-745.

Provencio, I., Loew, E.R., Foster, R.G., 1993. Vitamin A2-based photopigments within the pineal gland of a fully terrestrial vertebrates. Neurosci. Lett. 155, 223-226.

Radlwimmer, F.B., Yokoyama, S., 1998. The genetic analyses of the green visual pigments of rabbit (Oryctolagus cuniculus) and rat (Rattus norvegicus). Gene 218, 103-109.

Rafferty, C., Shichi, H., 1981. The involvement of water at the retinal binding site in rhodopsin and early light-induced intramolecular protein transfer. Photochem. Photobiol. 33, 229-234.

Sakmar, T.P., Franke, R.R., Khorana, H.G., 1989. Glutamic acid-113 serves as the retinylidene Schiff base counterion in bovine rhodopsin. Proc. Natl. Acad. Sci. USA 86, 8309-8313.

Schliewen, U., Fricke, H., Schertl, M., Eppen, J.T., Paabo, S., 1993. Which home for coelacanth? Nature 363, 405.

Shi, Y., Radlwimmer, F.B., Yokoyama, S., 2001. Molecular genetics and the evolution of ultraviolet vision in vertebrates. Proc. Natl. Acad. Sci. USA 98, 11731-11736.

Sun, H., Macke, J.P., Nathans, J., 1997. Mechanisms of spectral tuning in the mouse green cone pigment. Proc. Natl. Acad. Sci. USA 94, $8860-8865$.

Viitala, J., Korpimaki, E., Palokangas, P., Koivula, M., 1995. Attraction of kestrels to vole scent marks visible in ultraviolet light. Nature 373, $425-427$.

Vought, B.W., Dukkipatti, A., Max, M., Knox, B.E., Birge, R.R., 1999. Photochemistry of the primary event in short-wavelength visual opsins at low temperature. Biochemistry 38, 11287-11297.

Walls, G.L., 1934. The reptilian retina. Am. J. Ophthalmol. 17, 892-915.

Walls, G.L., 1942. The Vertebrate Eye and its Adaptive Radiation, Cranbrook Press, Bloomfield Hills, MI.

Wang, Z., Asenjo, A., Oprian, D.D., 1993. Identification of the $\mathrm{Cl}^{-}$-binding site in the human red and green color vision pigments. Biochemistry 32 , $2125-2130$.

Weitz, C.J., Nathans, J., 1993. Rhodospin activation: effect of the metarhodopsin I-metarhodopsin II equilibrium of neutralization or introduction of charged amino acids within putative transmembrane segments. Biochemistry 32, 14176-14182.
Whitmore, A.V., Bowmaker, J.K., 1989. Seasonal variation in cone sensitivity and short-wave absorbing visual pigments in the rudd Scadinius erythrophythalmus. J. Comp. Physiol. A 166, 103-115.

Wilkie, S.E., Robinson, P.R., Cronin, T.W., Poopalasundaram, S., Bowmaker, J.K., Hunt, D.M., 2000. Spectral tuning of avian violet- and ultraviolet-sensitive visual pigments. Biochemistry 39, 7895-7901.

Yang, Z., Kumar, S., Nei, M., 1997. A new method of inference of ancestral nucleotide and amino acid sequences. Genetics 141, 1641-1650.

Yokoyama, S., 1997. Molecular genetic basis of adaptive selection: Examples from color vision in vertebrates. Annu. Rev. Genet. 31, $315-336$.

Yokoyama, S., 2000a. Molecular evolution of vertebrate visual pigments. Prog. Ret. Eye Res. 19, 385-419.

Yokoyama, S., 2000b. Phylogenetic analysis and experimental approaches to study color vision in vertebrates. Methods Enzymol. 315, 312-325.

Yokoyama, S., Radlwimmer, F.B., 1998. The 'five-sites' rule and the evolution of red and green color vision in mammals. Mol. Biol. Evol. $15,560-567$.

Yokoyama, S., Radlwimmer, F.B., 1999. Molecular genetics of red and green color vision in mammals. Genetics 153, 919-932.

Yokoyama, S., Radlwimmer, F.B., 2001. The molecular genetics and evolution of red and green color vision in vertebrates. Genetics 158 , $1697-1710$.

Yokoyama, S., Shi, Y., 2000. Genetics and evolution of ultraviolet vision in vertebrates. FEBS Lett. 486, 167-172.

Yokoyama, S., Tada, T., 2000. Adaptation of the African and Indonesian coelacanths to deep-sea environments. Gene 261, 35-42.

Yokoyama, R., Yokoyama, S., 1990. Convergent evolution of the red- and green-like visual pigment genes in fish, Astyanax fasciatus, and human. Proc. Natl. Acad. Sci. USA 87, 9315-9318.

Yokoyama, S., Yokoyama, R., 1996. Adaptive evolution of photoreceptors and visual pigments in vertebrates. Annu. Rev. Ecol. Syst. 27, 543-567.

Yokoyama, R., Knox, B.E., Yokoyama, S., 1995. Rhodopsin from fish, Astyanax fasciatus: role of tyrosine 261 in the red shift. Invest. Ophthalmol. Vis. Res. 36, 939-945.

Yokoyama, S., Radlwimmer, F.B., Kawamura, S., 1998. Regeneration of ultraviolet pigments of vertebrates. FEBS Lett 423, 155-158.

Yokoyama, S., Zhang, H., Radlwimmer, F.B., Blow, N.S., 1999. Adaptive evolution of color vision of the Comoran coelacanth (Latimeria chalumnae). Proc. Natl. Acad. Sci. USA 96, 6279-6284.

Yokoyama, S., Radlwimmer, F.B., Blow, N.S., 2000. Ultraviolet pigments in birds evolved from violet pigments by a single amino acid change. Proc. Natl. Acad. Sci. USA 97, 7366-7371.

Zhukovsky, E.A., Oprian, D.D., 1989. Effect of carboxylic acid side chains on the absorption maximum of visual pigments. Science 246, 928-930. 